Check for updates

Cite this: RSC Adv., 2018, 8, 39777

Received 8th October 2018

Accepted 21st November 2018

DOI: $10.1039 / c 8 r a 08317 g$

rsc.li/rsc-advances

\section{Non-halogenated diphenyl-chalcogenide solvent processing additives for high-performance polymer bulk-heterojunction solar cells $\uparrow$}

\author{
Song Yi Park, (D) a Seyeong Song, (iD ${ }^{a}$ Yung Jin Yoon, (D) a Tack Ho Lee, (iD) \\ $\mathrm{Na}$ Gyeong An, (D) a Bright Walker (D)*b and Jin Young Kim (D) *a
}

\begin{abstract}
The ability to control the morphologies of active layers is a critical factor in the successful development of polymer solar cells (PSCs), and solvent processing additives offer a simple and effective way to accomplish this. In particular, diphenyl ether (DPE) is one of the most effective solvent additives but analogous additives based on this structure have not yet been extensively investigated. In this work, we have fabricated PSCs and investigated photovoltaic device characteristics using the series of non-halogenated, diphenyl-chalcogen solvent additives; DPE, diphenyl sulfide (DPS) and diphenyl selenide (DPSe). DPS devices showed optimal power conversion efficiencies (PCEs) of up to $9.08 \%$, and DPE devices also showed similarly high PCEs of up to $8.85 \%$. In contrast, DPSe devices showed relatively low PCEs (5.45\% at best) which we attribute to significant surface recombination and high series resistance, which led to limited open-circuit voltage $\left(V_{O C}\right)$. In the case of DPS, fast, field-independent photocurrent saturation with negligible bimolecular recombination led to efficient charge separation and collection, which resulted in the highest PCEs. Additionally, using 1,2,4-trimethylbenzene and DPS as an entirely non-halogenated solvent/additive system, we successfully demonstrated device fabrication with comparably high PCEs of up to $8.4 \%$. This work elucidates the effects of diphenyl-based solvent additives in PSCs and suggests a great potential of DPS as an effective non-halogenated solvent additive.
\end{abstract}

\section{Introduction}

Polymer : fullerene bulk-heterojunction solar cells (PSCs) have attracted great attention over the past two decades due to their many unique advantages such as their compatibility with simple and low-cost fabrication processes; large-area processability using scalable solution-based printing techniques; light-weight and mechanical flexibility. ${ }^{\mathbf{1 , 2}}$ Recently, high power conversion efficiencies (PCEs) over 11\%, which are comparable with amorphous silicon solar cells, have been reported using newly designed conjugated polymers and non-fullerene acceptors., ${ }^{3,4}$ High-performance PSCs can be achieved using several device fabrication strategies; incorporating effective electron or hole transport layers, ${ }^{5,6}$ via morphological control of bulkheterojunction (BHJ) active layers, ${ }^{7,8}$ using tandem structured devices, ${ }^{\mathbf{9}, 10}$ via photocurrent enhancement with surface plasmon resonance effects and so on. ${ }^{11-13}$ Among these strategies,

\footnotetext{
${ }^{a}$ Department of Energy Engineering, Ulsan National Institute of Science and Technology (UNIST), Ulsan 44919, South Korea. E-mail: jykim@unist.ac.kr

${ }^{b}$ Department of Chemistry, Kyung Hee University, Seoul 02447, South Korea. E-mail: walker@khu.ac.kr

$\dagger$ Electronic supplementary information (ESI) available: Crystalline packing properties (GIWAXS) and additional photovoltaic characteristics. See DOI: 10.1039/c8ra08317g
}

morphological control of the active layer is the most fundamentally important characteristic which influences device performance. It has been demonstrated that film morphology can be easily adjusted by using solvent processing additives such as 1,8-diiodooctane (DIO), 1,8-octanedithiol (ODT), 1-chloronaphthalene (CN), 1,2,4-trichlorobenzene (TCB), diphenyl ether (DPE), 2,3-dihydroxypyridine, 2,3-dihydroxypyridine etc., and these solvent additives help to enhance device performance., ${ }^{\mathbf{8 1 4 - 2 2}}$

So far, reports of high-performance PSCs have almost always involved optimization with halogenated solvents such as chlorobenzene (CB) and 1,2-dichlorobenzene, due to their excellent solvation of conjugated polymers. In addition, the most commonly reported solvent additives such as DIO, TCB and CN also include halogen atoms. However, halogenated solvents are hazardous to the natural environment and human health through either vapor or dermal exposure. Therefore, it would be highly desirable to use non-halogenated solvents to process PSCs, if possible.

Recently, environmental-friendly processed PSCs have been reported using anisole, 1,2,4-trimethylbenzene (TMB), 2-methylanisole, $o$-xylene and toluene as host solvent, and 1,2-dimethylnaphthalene and 1-phenylnaphthalene as solvent additives. ${ }^{23-27}$ With these non-halogenated solvent systems, the high PCE was achieved over $11 \%$ suggesting great potential of non-halogenated solvents. ${ }^{26}$ As non-halogenated solvent processed PSCs have attracted considerable attention recently, non-halogenated 
solvent processing additives merit further investigation for the continued development of environmentally friendly PSCs.

DPE is an excellent example of an effective non-halogenated solvent additive which promotes the formation of effective film morphologies and enhanced PCE values in a wide range of conjugated polymers. In particular, it is one of the most effective additives for use with semi-crystalline polymer based BHJs. For instance, poly[(2,5-bis-(2-hexyldecyloxy)phenylene)-alt-(5,6difluoro-4,7-di(thiophene-2-yl)benzo[c][1,2,5]-thiadiazole)] (PPDT2FBT) : [6:6]-phenyl-C ${ }_{71}$-butyric acid methyl ether $\left(\mathrm{PC}_{71} \mathrm{BM}\right)$ PSCs showed great PCE enhancement from $3.23 \%$ to $8.64 \%$ upon introduction of DPE. ${ }^{28}$ Furthermore, DPE is especially effective in thick active-layer PSCs, which produce larger short-circuit current densities $\left(J_{\mathrm{SC}}\right)$ through complete absorption of incident light, since it produces desirable morphologies throughout the whole film. ${ }^{29,30}$ Y. $\mathrm{Li}$ et al. reported $8 \%$ of PCE (with high $J_{\mathrm{SC}}=17.19 \mathrm{~mA} \mathrm{~cm}{ }^{-2}$ ) in $270 \mathrm{~nm}$ thick of poly(2,2': $5^{\prime}, 2^{\prime \prime}$-terthiophene-alt-2,3-bis(3,4-bis(octyloxy)phenyl)6,7-difluroquinoxaline) (PDFQx-3T) : $\mathrm{PC}_{71} \mathrm{BM}$ PSCs which showed well phase-separated film morphologies with low RMS roughness of $1.6 \mathrm{~nm}$ and low bimolecular recombination. ${ }^{31}$

Although DPE has been demonstrated as one of the most effective solvent additives discovered to date, a detailed understanding of why it is effective, and investigation of structurally related compounds is lacking. For instance, it might be expected that diphenyl sulfide (DPS) and diphenyl selenide (DPSe), which have similar molecular structures to DPE, may also be good candidates for non-halogenated solvent additives and yield improved device performance. Indeed, Y. Xia et al. reported that the PCE of a newly synthesized donor polymer: $\mathrm{PC}_{71} \mathrm{BM}$-based PSCs was greatly improved from $0.21 \%$ to $4.43 \%$ when DPS was used as a solvent additive. ${ }^{32}$ Although DPS showed outstanding properties as solvent additive, there are still no reports which thoroughly investigate the structure-property-function relationships for these additives (DPE, DPS and DPSe, so called DPX) to PSC performances.

In this work, the photovoltaic characteristics of poly [4,8-bis(5-(2-ethylhexyl)thiophen-2-yl)benzo[1,2- $\left.b: 4,5-b^{\prime}\right]$ dithiophene-co-3-fluorothieno[3,4- $b]$ thiophene-2-carboxylate] (PTB7$\mathrm{Th}): \mathrm{PC}_{71} \mathrm{BM}$ PSCs were investigated using the series of DPX additives with $\mathrm{CB}$ as the main solvent. Compared to control devices (without additives), all three DPX additives dramatically enhanced the short-circuit current density $\left(J_{\mathrm{SC}}\right)$ from 13.0 $\mathrm{mA} \mathrm{cm} \mathrm{cm}^{-2}$ to over $16.0 \mathrm{~mA} \mathrm{~cm}^{-2}$ due to desirable, phaseseparated film morphologies. The PSCs using DPS showed the best photovoltaic performance among the three solvent additives, with up to $9.08 \%$ PCE, while devices using DPE also showed high PCEs of $8.85 \%$. Devices with DPSe, however, exhibited significantly lower PCEs of $5.91 \%$ or less, largely due to low open-circuit voltages $\left(V_{\mathrm{OC}}\right)$ of only $0.68 \mathrm{~V}$ (compared to $\sim 0.80 \mathrm{~V}$ for the other additives). Analysis of the carrier dynamics in these devices reveals that the low PCE in DPSe devices originates from high surface recombination. Devices with DPS, however, exhibited negligible bimolecular recombination and efficient charge separation and extraction. Furthermore, DPX additives were investigated using TMB as a main solvent in a completely non-halogenated solvent system. PSCs with DPS additive showed excellent photovoltaic properties with excellent PCE $(8.24 \%)$, comparable to devices fabricated using CB. This report marks the first of its kind to provide a detailed, structure-function analysis of the class of additives based on the DPX topology to polymer BHJ films and photovoltaic device performances. Furthermore, PSCs with DPS exhibited the best device performances (PCE over 9\%), suggesting it can be useful to fabricate PSCs through environmental-friendly solution processes.

\section{Experimental section}

\section{General}

PTB7-Th and $\mathrm{PC}_{71} \mathrm{BM}$ were purchased from 1-Material and Organic Semiconductor Materials (OSM, Republic of Korea), respectively. Diphenyl ether and diphenyl sulfide were purchased from Sigma Aldrich and diphenyl selenide was purchased from Alfa Aesar. AFM height and phase images were obtained using a Veeco AFM microscope in tapping mode. Grazing incidence wide-angle X-ray scattering (GI-WAXS) measurements were carried out at the PLS-II 9A U-SAXS beam line of Pohang Accelerator Laboratory, Republic of Korea.

\section{Fabrication and characterization of PSCs}

PSC devices were prepared with a conventional structure of glass/ indium tin oxide (ITO)/poly(3,4-ethylenedioxythiophene):polystyrene sulfonate (PEDOT:PSS)/active layer/Al. Patterned ITO coated glass substrates were cleaned by ultra-sonication with deionized water, acetone and isopropyl alcohol. Then the substrates were dried in an oven at $100{ }^{\circ} \mathrm{C}$ overnight. PEDOT:PSS (Baytron, AI 4083) layers were spin coated on ITO substrates, and baked on a hot plate at $140{ }^{\circ} \mathrm{C}$ for $10 \mathrm{~min}$ in air. After baking, substrates were brought into a $\mathrm{N}_{2}$ filled glove box. For PTB7-Th : PC $_{71}$ BM $(1: 1.5, \mathrm{w} / \mathrm{w})$ PSCs, blend solutions were prepared in chlorobenzene $(\mathrm{CB})$ and 1,2,4-trimethylbenzene (TMB) with $3 \mathrm{vol} \%$ of diphenyl ether (DPE), diphenyl sulfide (DPS) and diphenyl selenide (DPSe) with total concentrations of $35 \mathrm{mg}$ $\mathrm{mL}^{-1}$ and $30 \mathrm{mg} \mathrm{mL}^{-1}$, respectively. The solutions were stirred at $60{ }^{\circ} \mathrm{C}$ overnight prior to spin casting. After coating active layers, substrates were brought into a high vacuum chamber $\left(\sim 10^{-6}\right.$ torr $)$, and $\mathrm{Al}(100 \mathrm{~nm})$ was deposited by thermal evaporation. Device areas were $13 \mathrm{~mm}^{2}$. Measurements were conducted in a nitrogen filled glove box using a high quality optical fiber to lead the light from a xenon arc lamp solar simulator. Intensity was calibrated at 100 $\mathrm{mW} \mathrm{cm}^{-2}$ with a standard silicon photodiode. Current densityvoltage $(J-V)$ characteristics were measured with a Keithley 2635A source measurement unit. Light intensity dependences of shortcircuit current density $\left(V_{\mathrm{SC}}\right)$ and open-circuit voltage $\left(V_{\mathrm{OC}}\right)$ were measured with neutral density filters. External quantum efficiency (EQE) was measured in the air using an EQE system (Model QEX7) by PV measurements Inc. (Boulder, Colorado).

\section{Results and discussion}

\section{Material properties}

To analyse the effects of DPX solvent additives on photovoltaic characteristics, we fabricated PSCs with high-performance $\mathrm{BHJ}$ 
active layer: PTB7-Th : $\mathrm{PC}_{71} \mathrm{BM}$. PTB7-Th is known as a semicrystalline conjugated polymer, and constitutes an appropriate candidate to check additive effects of DPX, since PTB7-Th has been widely studied as reference material with well-known properties, and also its semi-crystalline characteristics is appropriate for use with DPE. ${ }^{16,28}$ Detailed molecular structures and calculated dihedral angles (by DFT simulation) for the DPX series are shown in Fig. 1a and c, respectively. As the size the heteroatoms (' $\mathrm{X}$ ' in DPX) become larger, the dihedral angle increases from $34.2^{\circ}$ to $37.2^{\circ}$ to $47.5^{\circ}$ for DPE, DPS and DPSe, respectively. Furthermore, as the ' $\mathrm{X}$ ' atoms in DPX become heavier, the boiling point (at $760 \mathrm{mmHg}$ pressure) of each solvent increases; $258{ }^{\circ} \mathrm{C}, 296{ }^{\circ} \mathrm{C}$, and $335^{\circ} \mathrm{C}$ for DPE, DPS, and DPSe, respectively. Interestingly, the melting point of DPS occurs at the sub-zero temperature of $-40^{\circ} \mathrm{C}$, whereas DPE and DPSe have higher melting points of $26^{\circ} \mathrm{C}$ and $3{ }^{\circ} \mathrm{C}$, respectively. This stable liquid state of DPS facilitates device fabrication regardless of ambient temperatures in research laboratories or factories.

\section{Photovoltaic properties and morphology}

PSCs were fabricated in conventional structures with the architecture: indium tin oxide (ITO)/poly(3,4ethylenedioxythiopene) polystyrene sulfonate (PEDOT:PSS)/ PTB7-Th : $\mathrm{PC}_{71} \mathrm{BM} / \mathrm{Al}$. A corresponding energy band diagram is shown in Fig. 1b. For the active layer (PTB7-Th : $\mathrm{PC}_{71} \mathrm{BM}$ ), CB was used as the main solvent and 3 vol\% of DPX processing additives were added. Detailed device fabrication procedures are described in the Experimental section. Photovoltaic properties of the PSCs were investigated by comparison with control devices (only CB used) and CB +3 vol\% DPX devices. Corresponding current density-voltage $(J-V)$ curves and external quantum efficiency (EQE) spectra are shown in Fig. 2a and b, respectively. Detailed photovoltaic parameters are described in Table 1. Control devices with no additives showed low PCEs of $4.98 \%$ or less, whereas devices processed with DPX showed higher PCEs of $8.85 \%, 9.08 \%$ and $5.91 \%$ for DPE, DPS and

(a)

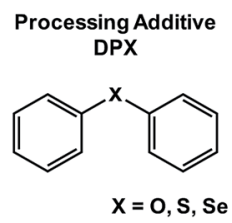

(c)

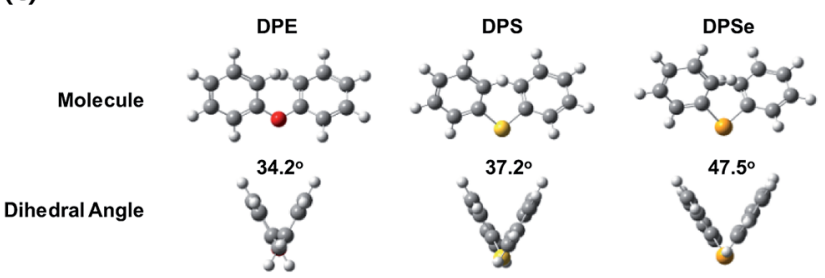

Fig. 1 (a) Molecular structure of DPX processing additives. (b) Energy band diagram of PTB7-Th: PC $_{71}$ BM PSCs. (c) Detailed molecular structure and dihedral angles of DPX additives calculated by DFT. (a)

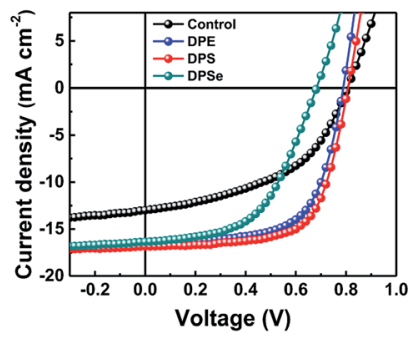

(c)

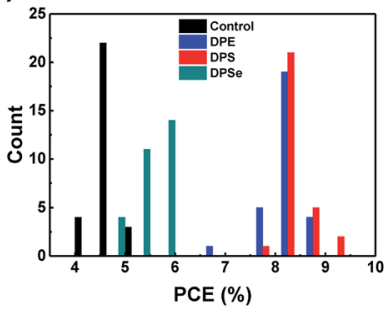

(b)

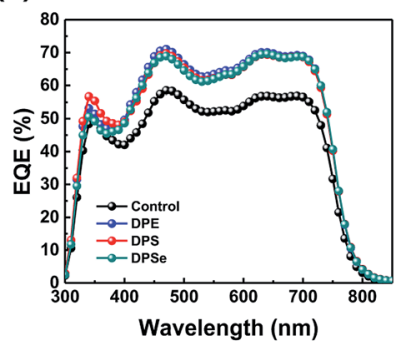

(d)

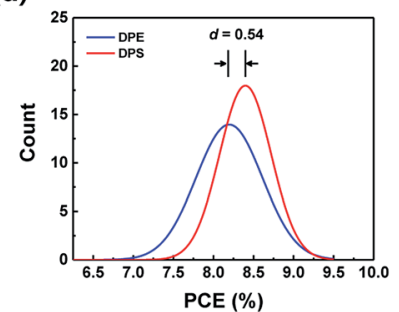

Fig. 2 (a) J-V characteristics, (b) EQE spectra and (c) statistical histogram of PTB7-Th : PC ${ }_{71}$ BM PSCs with different solvent additives. (d) Normal distribution curve for PCE values with comparison between DPE and DPS devices. ( $d$ is calculated as Cohen's $d$ which is a statistical term.)

DPSe, respectively. Except for DPSe devices, both DPE and DPS devices showed excellent performance. In particular, DPS devices exhibited the best photovoltaic device performance including a $J_{\mathrm{SC}}$ of $16.8 \mathrm{~mA} \mathrm{~cm}^{-2}, V_{\mathrm{OC}}$ of $0.81 \mathrm{~V}$ and $\mathrm{FF}$ of 0.67 , leading a PCE over 9\%. Furthermore, as shown in statistical distribution of PCE as shown in Fig. 2c, the average PCE $\left(\mathrm{PCE}_{\mathrm{avg}}\right)$ of DPS devices is around $8.4 \%$ while $\mathrm{PCE}_{\mathrm{avg}}$ of DPE devices was around $8.2 \%$. In order to determine whether the observed increase in PCE is statistically meaningful, we calculated Cohen's $d$ value, which is defined as the difference between two means divided a common standard deviation for the data sets, providing a quantitative difference for specific phenomenon between two independent groups. ${ }^{33}$ Since the difference between $\mathrm{PCE}_{\mathrm{avg}}$ of DPE and DPS devices is relatively small (around $0.2 \%$ ), the significance of this difference can be ascertained by calculating Cohen's $d$. If Cohen's $d$ is $0.2,0.5$ or 0.8 , this corresponds to small, medium or large effects, respectively. As shown in Fig. 2d, calculated Cohen's $d$ for devices prepared with DPE vs. DPS is 0.54 indicating a moderate effect, or greater than $69.1 \%$ confidence that devices processed with DPS yield higher average performance than DPE. This small but statistically significant increase in PCE in DPS devices (compared to DPE devices) is related to reduced bimolecular recombination, which will be discussed subsequently. Compared to DPE and DPS devices, the $J_{\mathrm{SC}}$ of DPSe devices was a similar value of $16 \mathrm{~mA} \mathrm{~cm}{ }^{-2}$ however, the $V_{\mathrm{OC}}$ and $\mathrm{FF}$ were significantly lower at $0.68 \mathrm{~V}$ and 0.53 , respectively. The reason for low $V_{\mathrm{OC}}$ and FF in DPSe devices may originate from surface recombination or traps which render DPSe an ineffective processing additive, despite an improved $J_{\mathrm{SC}}$.

To investigate the influence of processing additives on film morphologies in detail, we first analysed the molecular ordering and packing structures of PTB7-Th : $\mathrm{PC}_{71} \mathrm{BM}$ blend films via 
Table 1 Summary of photovoltaic parameters of PTB7-Th : PC71BM PSCs processed with different solvent additives

\begin{tabular}{|c|c|c|c|c|c|}
\hline Solvent & Solvent additive (3 vol\%) & $J_{\mathrm{SC}}\left[\mathrm{mA} \mathrm{cm}^{-2}\right]$ & $V_{\mathrm{OC}}[\mathrm{V}]$ & $\mathrm{FF}$ & PCE $[\%]$ \\
\hline \multirow[t]{3}{*}{$\mathrm{CB}^{a}$} & Control & $13.0(12.4 \pm 0.51)$ & $0.80(0.79 \pm 0.02)$ & $0.48(0.48 \pm 0.01)$ & $4.98(4.80 \pm 0.24)$ \\
\hline & DPE & $16.5(15.6 \pm 0.61)$ & $0.80(0.80 \pm 0.01)$ & $0.67(0.66 \pm 0.02)$ & $8.85(8.20 \pm 0.41)$ \\
\hline & DPSe & $16.4(14.8 \pm 0.75)$ & $0.68(0.70 \pm 0.03)$ & $0.53(0.53 \pm 0.03)$ & $5.91(5.45 \pm 0.34)$ \\
\hline \multirow[t]{2}{*}{$\mathrm{TMB}^{b}$} & Control & $14.9(14.0 \pm 0.46)$ & $0.76(0.76 \pm 0.01)$ & $0.53(0.53 \pm 0.02)$ & $6.06(5.61 \pm 0.32)$ \\
\hline & DPE & $16.5(15.3 \pm 0.65)$ & $0.78(0.78 \pm 0.00)$ & $0.62(0.63 \pm 0.01)$ & $8.02(7.50 \pm 0.26)$ \\
\hline
\end{tabular}

${ }^{a}$ Average values obtained from 30 devices are stated in parentheses. ${ }^{b}$ Average values obtained from 10 devices are stated in parentheses.

grazing incidence wide-angle X-ray scattering (GIWAXS). As shown in Fig. S1, $\dagger$ all of the film processing conditions showed negligible differences in GIWAXS pattern images and line-cut profiles, except for a slight enhancement of the (100) peak intensity for DPX films along the $q_{z}$ direction. This indicates that DPX does not strongly affect the molecular ordering of BHJ films, although (100) lamellar interactions in the $q_{z}$ direction were slightly strengthened by DPX additives.

We also analysed PTB7-Th : $\mathrm{PC}_{71} \mathrm{BM}$ blend films via atomic force microscopy (AFM), in order to characterize the surface morphologies. As shown in Fig. 3, a lot of aggregated features with large domain size $(\sim 100 \mathrm{~nm})$ were observed for control films with no additives, and this large domains might be attributed to the fullerene-rich regions. ${ }^{34}$ These control films exhibited an RMS roughness of $3.43 \mathrm{~nm}$; the morphologies and roughness are consistent with previously reported results for PTB7-Th : $\mathrm{PC}_{71} \mathrm{BM}$ BHJs. ${ }^{34,35}$ However, upon using DPX additives, the aggregation was significantly reduced with decreased RMS roughness of $1.55 \mathrm{~nm}, 1.75 \mathrm{~nm}$ and $1.81 \mathrm{~nm}$ for DPE, DPS and DPSe, respectively. Furthermore, relatively small aggregated features were evenly distributed throughout the film compared to control films, which suggests that the surface area between donor and acceptor phases is larger for all films processed with DPX additives, which is consistent with the increase $J_{\mathrm{SC}} \mathrm{S}$ from $13 \mathrm{~mA} \mathrm{~cm}{ }^{-2}$ to more than $16 \mathrm{~mA} \mathrm{~cm}^{-2}$ upon processing with all three DPX additives. Therefore, DPX additives facilitated the formation of well phase-separated film morphologies without changing intermolecular ordering and packing properties, a phenomenon which has been documented in previous studies. ${ }^{28}$

\section{Charge carrier recombination and transport properties}

To elucidate the mechanisms of charge recombination in PSCs processed with and without DPX additives, light intensity $\left(I_{\text {light }}\right)$ dependent $J_{\mathrm{SC}}$ and $V_{\mathrm{OC}}$ values were measured. In general, $J_{\mathrm{SC}}$ follows a power law of $J_{\mathrm{SC}} \propto I_{\text {light }}$, where $I_{\text {light }}$ is the incident light intensity and $s$ is an exponent. As $s$ approaches unity, this indicates that bimolecular recombination rates approach zero, whereas decreasing values of $s$ indicate increasing rates of bimolecular recombination. ${ }^{36}$ As shown in Fig. 4a, the control device with no additive showed the lowest $s$ value of 0.941 , which is consistent with poor photovoltaic performance. For devices processed with DPX additives, DPS yielded the highest $s$ value among the three additives of 0.985 , indicating that it has the greatest ability to reduce bimolecular recombination, resulting in excellent PSC performance. DPE also showed a similar $s$ value of 0.980 . However, in DPSe devices, a significantly lower $s$ value of 0.970 was obtained, indicating that the poor device performance relative to DPS and DPE originates, in part, from higher bimolecular recombination rates.

If bimolecular recombination is the only loss mechanism in a given $\mathrm{BHJ}$ system, the $V_{\mathrm{OC}}$ follows eqn (1);

$$
V_{\mathrm{OC}}=\frac{E_{\text {gap }}}{q}-\frac{k T}{q} \ln \left[\frac{\left(1-P_{\mathrm{D}}\right) \gamma N_{\mathrm{C}}^{2}}{P_{\mathrm{D}} G}\right]
$$

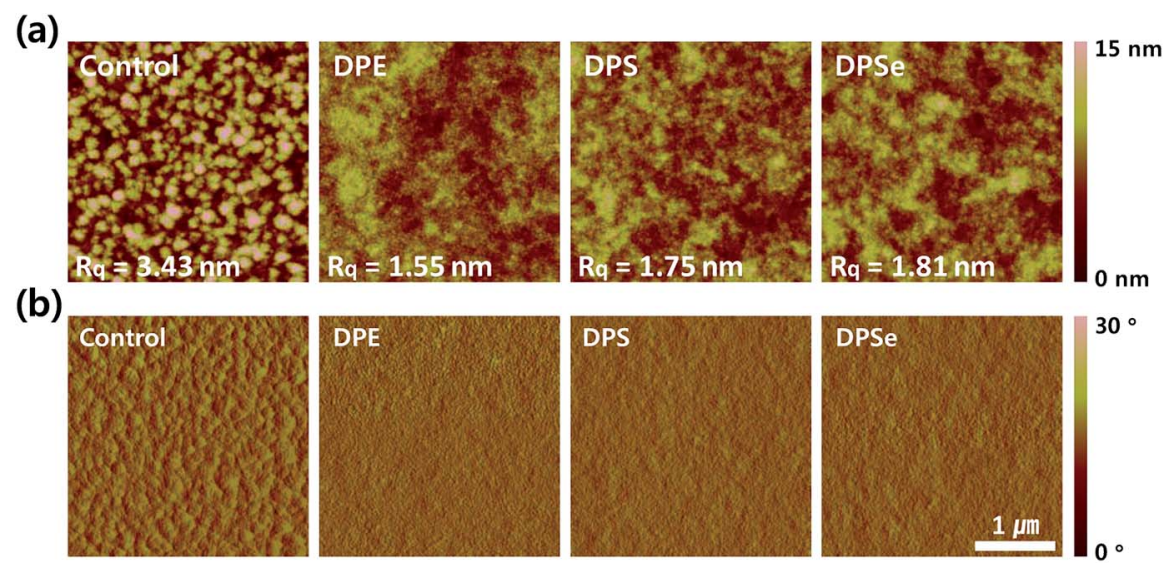

Fig. 3 AFM (a) topographical and (b) phase images for PTB7-Th : PC ${ }_{71}$ BM blend films processed with different solvent additives. 
(a)

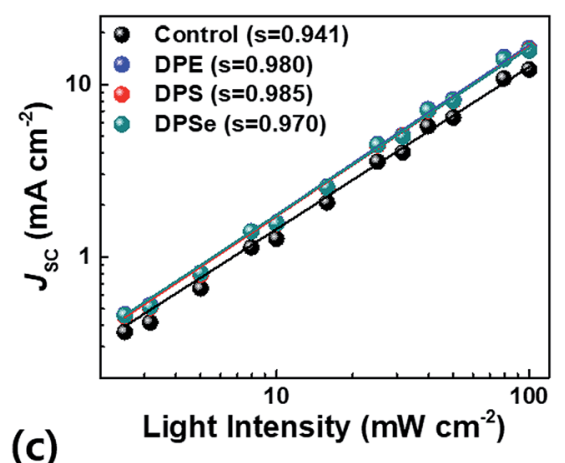

(c)

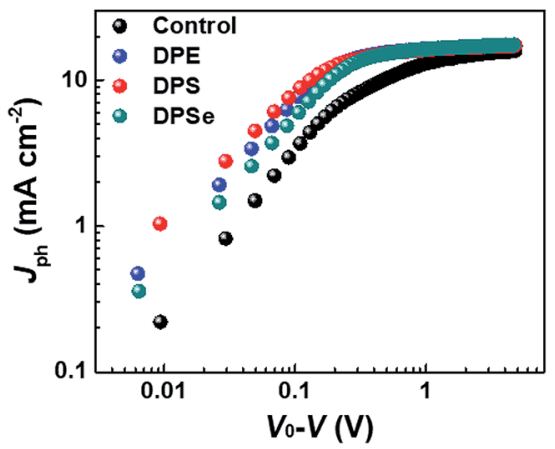

(b)
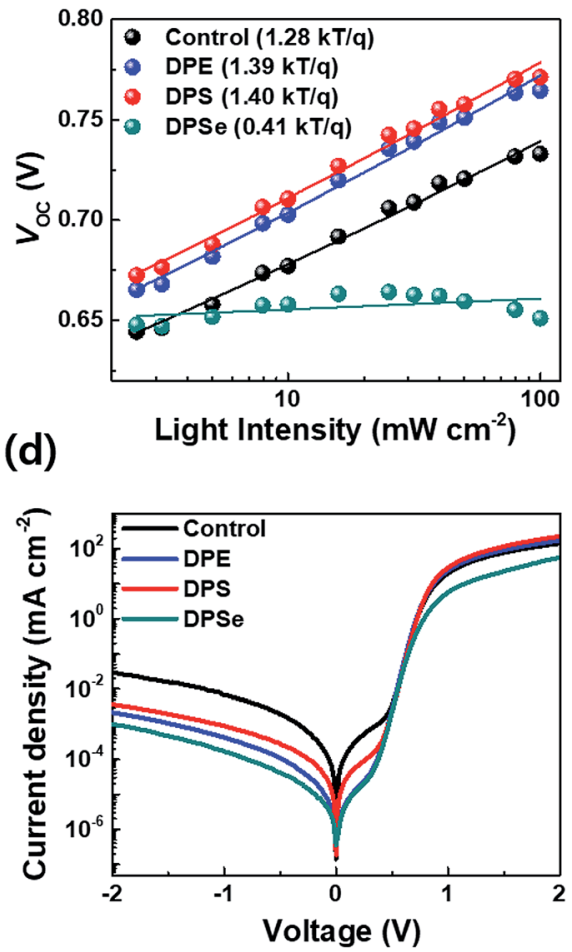

Fig. 4 Light intensity dependent (a) $J_{S C}$ and (b) $V_{\text {OC }}$ of PTB7-Th : $P C_{71} B M$ PSCs. (c) Photocurrent density $\left(J_{\text {ph }}\right)-$ effective voltage $\left(V_{\text {eff }}=V_{0}-V\right)$ and $(d)$ dark current density $\left(J_{D}\right)$-voltage $(V)$ characteristics for PTB7-Th : PC ${ }_{71} B M$ PSCs.

where $E_{\text {gap }}$ is the energy difference between the $\mathrm{HOMO}_{\text {donor }}$ and $\mathrm{LUMO}_{\text {acceptor }}, q$ is the elementary charge, $k$ is the Boltzmann constant, $T$ is temperature in Kelvin, $P_{\mathrm{D}}$ is the dissociation probability of the electron (e)-hole (h) pairs, $\gamma$ is the Langevin recombination constant, $N_{\mathrm{C}}$ is the effective density of states, and $G$ is the generation rate of bound e-h pairs. Since $G$ is the only parameter directly proportional to $I_{\text {light }}$, we can obtain information about the existence of additional trap-assisted recombination from $V_{\text {OC }} v s$. $\ln \left(I_{\text {light }}\right)$ plots. ${ }^{36,37}$ As shown in Fig. $4 \mathrm{~b}$, control devices showed a slope of $1.28 \mathrm{kT} / \mathrm{q}$, while the slope values for DPE, DPS and DPSe devices were observed to be $1.39,1.40$ and $0.41 \mathrm{kT} / q$, respectively. Although the slope value of control devices was closest to $k T / q$ (which indicates the lowest rate of trap-assisted recombination among all conditions), considering these results together with $J_{\mathrm{SC}} v s$. $I_{\text {light }}$, indicates that the combination of relatively high rates of bimolecular recombination and low rates of trap-assisted recombination together resulted overall poor device performance. Similarly, although DPE and DPS devices showed slightly higher trapassisted recombination compared to control devices, their negligible bimolecular recombination leads to superior device performance. In the case of DPSe devices, however, an unusual slope value lower than $k T / q$ was observed which indicates a high rate of surface recombination, rather than trap-assisted recombination, leading to a limited $V_{\text {OC }}{ }^{38-40}$

To investigate the location of surface recombination in the devices, we fabricate devices with the configuration ITO/ PEDOT:PSS/3\% DPSe/active layer (3\% DPE)/Al, where 3\% DPSe indicates spin-coating of CB +3 vol\% DPSe solvents on the
PEDOT:PSS layer. As shown in Fig. S2 and Table S1, $\dagger$ this device showed almost identical device characteristics to those processed with DPSe in the active layer; therefore we consider that the surface recombination in DPSe devices occurs at the boundary between PEDOT:PSS and the active layer. Furthermore, due to the high boiling point of DPSe, the residual additive in $\mathrm{BHJ}$ films may lead to poor device performance. To investigate this possibility, we fabricated PSCs with methanol a $(\mathrm{MeOH})$ washing treatment, which can remove residual solvent additives due to the insolubility of the active layer in $\mathrm{MeOH}$ but miscibility with additives; this allows residual additives to be extracted without adversely affecting the active layer morphology. ${ }^{41}$ As shown in Fig S2 and Table S2, $\dagger$ after $\mathrm{MeOH}$ treatment, the $J-V$ curves and efficiency of DPSe processed devices were recovered, showing PCE (8.39\%) comparable to DPE and DPS devices. Therefore, we surmise that residual DPSe in $\mathrm{BHJ}$ films affects not only surface recombination, but may also disturb charge transport.

Photocurrent density-effective voltage $\left(J_{\mathrm{ph}}-V_{\text {eff }}\right)$ characteristics were collected to investigate charge generation and extraction properties, as shown in Fig. 4c. In control devices, $J_{\mathrm{ph}}$ became saturated at a high $V_{\text {eff }}$ of greater than $1 \mathrm{~V}$, indicating that a high electric-field was needed to completely separate bound $\mathrm{e}-\mathrm{h}$ pairs and extract separated charge carriers. In contrast, DPS devices showed rapid $J_{\text {ph }}$ saturation at a low $V_{\text {eff }}$ of around $0.19 \mathrm{~V}$, while $J_{\mathrm{ph}}$ saturation in DPE and DPSe devices occurred at $V_{\text {eff }}$ values of around 0.21 and $0.25 \mathrm{~V}$, respectively. The fast and field-independent $J_{\mathrm{ph}}$ saturation in DPS devices suggests negligible recombination losses and efficient charge 
collection under operating conditions. Dark current densityvoltage $\left(J_{\mathrm{D}}-V\right)$ characteristics were measured as shown in Fig. 4d. Shunt and series resistance ( $R_{\mathrm{sh}}$ and $R_{\mathrm{s}}$, respectively) values calculated from $J_{\mathrm{D}}-V$ curve are summarized in Table $\mathrm{S} 4 \dagger$ as well. Control devices showed poor diode properties due to high leakage currents and low $R_{\mathrm{sh}}$. For DPX devices, leakage currents in the shunt regime were an order of magnitude lower than control devices, consistent with high $J_{\mathrm{SC}} \mathrm{S}$. However, DPSe showed the highest $R_{\mathrm{S}}$ of $13.5 \Omega \mathrm{cm}^{2}$ among three DPX devices; this high $R_{\mathrm{S}}$ is related to excessive surface recombination, and leads to poor photovoltaic device performance. ${ }^{40}$

To confirm that the three DPX solvent additives are effective in non-halogenated host solvents (as opposed to CB), we fabricated PSCs using fully non-halogenated solvent:additive systems based on 1,2,4-trimethylbenzene (TMB) as the primary solvent with the three DPX compounds as solvent additives. Corresponding $J-V$ curves and EQE spectra are shown in Fig. 5 and summary of photovoltaic parameters is described in Table 1. Among all conditions, DPS devices again yielded the highest PCEs of up to $8.24 \%$, with corresponding $J_{\mathrm{SC}}$ of $16.1 \mathrm{~mA} \mathrm{~cm}^{-2}$, $V_{\text {OC }}$ of $0.79 \mathrm{~V}$ and FF of 0.65. DPE devices also showed high PCEs of up to $8.02 \%$, whereas control devices and DPSe devices showed lower PCEs of $6.06 \%$ and $5.08 \%$, respectively. The consistently outstanding device performance observed in DPS devices fabricated with both $\mathrm{CB}$ and TMB as primary solvents confirms the great potential of DPS for PSC fabrication in research laboratories and in industry.

\section{(a)}

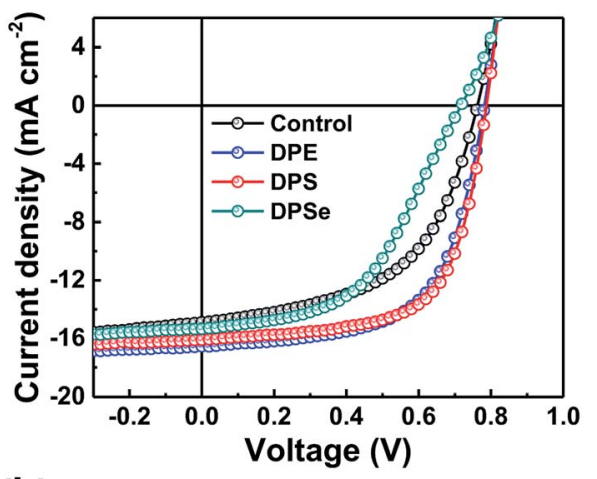

(b)

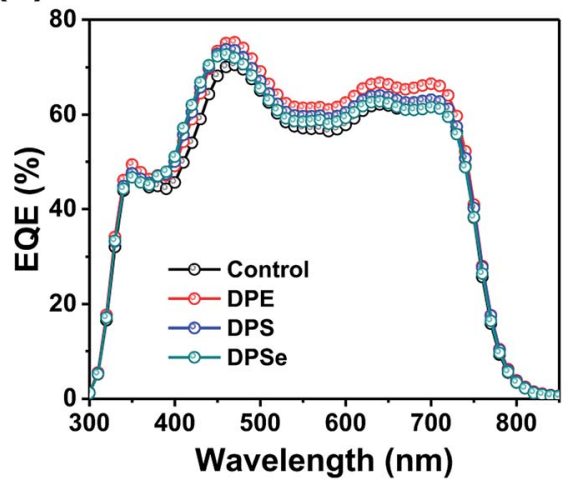

Fig. 5 (a) J-V characteristics and (b) EQE spectra for PTB7Th: $\mathrm{PC}_{71} \mathrm{BM}$ PSCs using 1,2,4-trimethylbenzene as a primary solvent.

\section{Conclusions}

In conclusion, we have investigated the photovoltaic characteristics, film morphologies and device properties of PTB7Th : PC $_{71}$ BM PSCs using DPE, DPS and DPSe solvent additives. Compared to control devices without additives, all three solvent additives provided increased $J_{\mathrm{SC}} \mathrm{S}$ of over $16 \mathrm{~mA} \mathrm{~cm}{ }^{-2}$. This enhancement is attributed to well-phase-separated film morphologies with low RMS roughness $(\sim 1.5 \mathrm{~nm})$. PSCs with DPS consistently yielded the best performance among three additives, with PCEs of great than 9\%, a small but significant improvement compared to DPE devices, which also showed high PCEs of up to $8.85 \%$. In contrast, DPSe devices showed relatively low PCEs of $5.91 \%$ or less, largely due to a low $V_{\mathrm{OC}}$, which originated from significant surface recombination and high series resistance. For DPS devices, quick and fieldindependent $J_{\mathrm{ph}}$ saturation with negligible bimolecular recombination occurred, leading to efficient charge separation and collection and excellent device performance. Finally, we successfully demonstrated the fabrication of PSCs using fully non-halogenated solvent : additive systems incorporating TMB as a primary solvent and DPX materials as solvent additives. Using TMB as the main solvent, DPS devices showed excellent PCEs of up to $8.24 \%$, while DPE devices also showed high PCEs of up to $8.02 \%$, while DPSe devices showed low PCEs of $\sim 5 \%$. We confirmed that DPS is the most effective solvent additive for use in PSCs by comparison of the three diphenyl chalcogenide additives. This work demonstrates the suitability of DPS as an effective non-halogenated solvent additive for PSC fabrication, offering the possibility of safe and stable solution-processing using non-halogenated solvents in ambient conditions considerations which are greatly needed for the industrial development and commercialization of PSCs.

\section{Conflicts of interest}

There are no conflicts to declare.

\section{Acknowledgements}

This research was supported by the National Research Foundation of Korea (No. NRF-2017R1C1B1010627, 2015M1A2A2057506, and 2016M1A2A2940914) and Kyung Hee University new researcher fund (No. 20180931).

\section{Notes and references}

1 M. Kaltenbrunner, M. S. White, E. D. Głowacki, T. Sekitani, T. Someya, N. S. Sariciftci and S. Bauer, Nat. Commun., 2012, 3, 770.

2 F. C. Krebs, S. A. Gevorgyan and J. Alstrup, J. Mater. Chem., 2009, 19, 5442-5451.

3 Q. Fan, Y. Wang, M. Zhang, B. Wu, X. Guo, Y. Jiang, W. Li, B. Guo, C. Ye, W. Su, J. Fang, X. Ou, F. Liu, Z. Wei, T. C. Sum, T. P. Russell and Y. Li, Adv. Mater., 2018, 30, 1704546. 
4 W. Zhao, D. Qian, S. Zhang, S. Li, O. Inganäs, F. Gao and J. Hou, Adv. Mater., 2016, 28, 4734-4739.

5 X. Ouyang, R. Peng, L. Ai, X. Zhang and Z. Ge, Nat. Photonics, 2015, 9, 520.

6 K. Zhang, Z. Hu, R. Xu, X. F. Jiang, H. L. Yip, F. Huang and Y. Cao, Adv. Mater., 2015, 27, 3607-3613.

7 Y. Liu, J. Zhao, Z. Li, C. Mu, W. Ma, H. Hu, K. Jiang, H. Lin, H. Ade and H. Yan, Nat. Commun., 2014, 5, 5293.

8 J. Peet, J. Y. Kim, N. E. Coates, W. L. Ma, D. Moses, A. J. Heeger and G. C. Bazan, Nat. Mater., 2007, 6, 497.

9 J. Y. Kim, K. Lee, N. E. Coates, D. Moses, T.-Q. Nguyen, M. Dante and A. J. Heeger, Science, 2007, 317, 222-225.

10 S. Song, K. Kranthiraja, J. Heo, T. Kim, B. Walker, S. H. Jin and J. Y. Kim, Adv. Energy Mater., 2017, 7, 1700782.

11 H. Choi, S.-J. Ko, Y. Choi, P. Joo, T. Kim, B. R. Lee, J.-W. Jung, H. J. Choi, M. Cha, J.-R. Jeong, I.-W. Hwang, M. H. Song, B.-S. Kim and J. Y. Kim, Nat. Photonics, 2013, 7, 732.

12 S. Song, J. Heo, T. K. Lee, S. Park, B. Walker, S. K. Kwak and J. Y. Kim, J. Phys. Chem. C, 2017, 121, 17569-17576.

13 J.-L. Wu, F.-C. Chen, Y.-S. Hsiao, F.-C. Chien, P. Chen, C.-H. Kuo, M. H. Huang and C.-S. Hsu, ACS Nano, 2011, 5, 959-967.

14 C. V. Hoven, X. D. Dang, R. C. Coffin, J. Peet, T. Q. Nguyen and G. C. Bazan, Adv. Mater., 2010, 22, E63-E66.

15 L. Dou, J. Gao, E. Richard, J. You, C.-C. Chen, K. C. Cha, Y. He, G. Li and Y. Yang, J. Am. Chem. Soc., 2012, 134, 10071-10079.

16 T. L. Nguyen, H. Choi, S. J. Ko, M. A. Uddin, B. Walker, S. Yum, J. E. Jeong, M. H. Yun, T. J. Shin, S. Hwang, J. Y. Kim and H. Y. Woo, Energy Environ. Sci., 2014, 7, 3040-3051.

17 T. H. Lee, M. A. Uddin, C. Zhong, S. J. Ko, B. Walker, T. Kim, Y. J. Yoon, S. Y. Park, A. J. Heeger, H. Y. Woo and J. Y. Kim, Adv. Energy Mater., 2016, 6, 1600637.

18 S. Lee, J. Kong and K. Lee, Adv. Energy Mater., 2016, 6, 1600970.

19 G. Sai-Anand, A. Dubey, A.-I. Gopalan, S. Venkatesan, S. Ruban, K. M. Reza, J. Choi, K. S. Lakhi, B. Xu, Q. Qiao and A. Vinu, Sol. Energy Mater. Sol. Cells, 2018, 182, 246-254.

20 B. Xu, G. Sai-Anand, A.-I. Gopalan, Q. Qiao and S.-W. Kang, Polymers, 2018, 10, 121.

21 S. Venkatesan, J. Chen, E. C. Ngo, A. Dubey, D. Khatiwada, C. Zhang and Q. Qiao, Nano Energy, 2015, 12, 457-467.

22 S. Venkatesan, N. Adhikari, J. Chen, E. C. Ngo, A. Dubey, D. W. Galipeau and Q. Qiao, Nanoscale, 2014, 6, 1011-1019.
23 C.-C. Chueh, K. Yao, H.-L. Yip, C.-Y. Chang, Y.-X. Xu, K.-S. Chen, C.-Z. Li, P. Liu, F. Huang, Y. Chen, W.-C. Chen and A. K. Y. Jen, Energy Environ. Sci., 2013, 6, 3241-3248.

24 S. Venkatesan, Q. Chen, E. C. Ngo, N. Adhikari, K. Nelson, A. Dubey, J. Sun, V. Bommisetty, C. Zhang, D. Galipeau and Q. Qiao, Energy Technol., 2014, 2, 269-274.

25 H. Zhang, H. Yao, W. Zhao, L. Ye and J. Hou, Adv. Energy Mater., 2016, 6, 1502177.

26 J. Zhao, Y. Li, G. Yang, K. Jiang, H. Lin, H. Ade, W. Ma and H. Yan, Nat. Energy, 2016, 1, 15027.

27 Y. Wu, Y. Zou, H. Yang, Y. Li, H. Li, C. Cui and Y. Li, ACS Appl. Mater. Interfaces, 2017, 9, 37078-37086.

28 T. H. Lee, S. Y. Park, B. Walker, S.-J. Ko, J. Heo, H. Y. Woo, H. Choi and J. Y. Kim, RSC Adv., 2017, 7, 7476-7482.

29 S. J. Ko, B. Walker, T. L. Nguyen, H. Choi, J. Seifter, M. A. Uddin, T. Kim, S. Kim, J. Heo, G. H. Kim, S. Cho, A. J. Heeger, H. Y. Woo and J. Y. Kim, Adv. Funct. Mater., 2016, 26, 3324-3330.

30 Y. Zheng, T. Goh, P. Fan, W. Shi, J. Yu and A. D. Taylor, ACS Appl. Mater. Interfaces, 2016, 8, 15724-15731.

31 Y. Li, S.-J. Ko, S. Y. Park, H. Choi, T. L. Nguyen, M. A. Uddin, T. Kim, S. Hwang, J. Y. Kim and H. Y. Woo, J. Mater. Chem. A, 2016, 4, 9967-9976.

32 Y. Xia, H. Zhang, J. Li, J. Tong, P. Zhang and C. Yang, J. Polym. Res., 2014, 22, 633.

33 J. Cohen, Statistical Power Analysis for the Behavioral Sciences, Lawrence Erlbaum Associates, New York, 2nd edn, 1988.

34 J. Kong, I. W. Hwang and K. Lee, Adv. Mater., 2014, 26, 62756283.

35 L. Zhao, S. Zhao, Z. Xu, Q. Yang, D. Huang and X. Xu, Nanoscale, 2015, 7, 5537-5544.

36 A. K. K. Kyaw, D. H. Wang, V. Gupta, W. L. Leong, L. Ke, G. C. Bazan and A. J. Heeger, ACS Nano, 2013, 7, 4569-4577.

37 L. J. A. Koster, V. D. Mihailetchi, R. Ramaker and P. W. M. Blom, Appl. Phys. Lett., 2005, 86, 123509.

38 V. V. Brus, Org. Electron., 2016, 29, 1-6.

39 N. Bauer, Q. Zhang, J. Zhao, L. Ye, J.-H. Kim, I. Constantinou, L. Yan, F. So, H. Ade, H. Yan and W. You, J. Mater. Chem. A, 2017, 5, 4886-4893.

40 S. Y. Park, Y. Li, J. Kim, T. H. Lee, B. Walker, H. Y. Woo and J. Y. Kim, ACS Appl. Mater. Interfaces, 2018, 10, 3885-3894.

41 L. Ye, Y. Jing, X. Guo, H. Sun, S. Zhang, M. Zhang, L. Huo and J. Hou, J. Phys. Chem. C, 2013, 117, 14920-14928. 